\title{
Bias in spatial memory: a categorical endorsement
}

\author{
Daniel B.M. Haun a,b,c,*, Gary L. Allen ${ }^{\text {a }}$, Douglas H. Wedell ${ }^{\text {a }}$ \\ a Department of Psychology, University of South Carolina, Barnwell College, Columbia, \\ SC 29208, United States \\ ${ }^{\mathrm{b}}$ Max Planck Institute for Psycholinguistics, Wundtlaan 1, PB 310, 6500 AH Nijmegen, The Netherlands \\ ${ }^{\mathrm{c}}$ F.C. Donders Centre for Cognitive Neuroimaging, PB 9101, 6500 HB Nijmegen, The Netherlands
}

Available online 24 November 2004

\begin{abstract}
Two experiments investigated how angular estimates reflect bias as a function of response mode, geometric plane of variation, number of implicit categories, memory load and intervening task conditions. In Experiment 1, participants made motor and verbal estimates of incline and azimuth from memory. Estimates in both response modes showed signs of bias predicted by a single-category adaptation of Huttenlocher et al. [Huttenlocher, J., Hedges, L. V., \& Duncan, S. (1991). Categories and particulars: Prototype effects in estimating spatial location. Psychological Review, 98, 352-376] category-adjustment model. In Experiment 2, participants made motor estimates of azimuth from memory under a variety of conditions. Stimuli in this experiment were distributed along two contiguous spatial categories. Although increasing levels of cognitive load did not produce a graded effect, participants' estimates were biased and were well described by a multiple-category adaptation of the category-adjustment model. Results from both studies supported an implicit region-based model of bias in spatial memory. These findings were discussed with respect to accounts of spatial memory that propose multiple systems or formats for coding.
\end{abstract}

(c) 2004 Elsevier B.V. All rights reserved.

\footnotetext{
${ }^{*}$ Corresponding author at: Max Planck Institute for Psycholinguistics, Wundtlaan 1, PB 310, 6500 AH Nijmegen, The Netherlands.

E-mail address: daniel.haun@mpi.nl (D.B.M. Haun).
} 


\section{Introduction}

Human spatial memory is both accurate and biased, a paradox that poses an interesting challenge for researchers studying spatial cognition and behavior. Accuracy is reflected in the precision of memory-directed responses to objects and surfaces (Creem \& Proffitt, 1998; Lee, 1993) and in the geometric veridicality of place memory (O'Keefe \& Nadel, 1978; Waller, Loomis, Golledge, \& Beall, 2000; Hartley, Trinkler, $\&$ Burgess, 2004). Bias is reflected in the systematic distortion of recollected location (Allen, 1981; Stevens \& Coupe, 1978; Huttenlocher, Hedges, \& Duncan, 1991) and estimated incline (Creem \& Proffitt, 1998). Contemporary accounts of these phenomena often involve either two systems or two processes, distinct but interactive (Allen \& Haun, 2004).

The distinction between two systems or processes for coding spatial relations is based empirically on evidence showing that motor responses are more precise and less biased than verbal estimates of the same spatial relations, which show greater error and variability (e.g., Bhalla \& Proffitt, 1999, 2000; Creem \& Proffitt, 1998, 1999, 2001a, 2001b; Eby \& Loomis, 1987; Loomis, Silva, Fujita, \& Fukusima, 1992; Philbeck \& Loomis, 1997; Wraga, Creem, \& Proffitt, 2000). While spatial bias towards explicit visual landmarks has been found early in motor responses, as well (Diedrichsen, Werner, Schmidt, \& Trommershäuser, 2004; Werner \& Diedrichsen, 2002), such estimates are again more accurate and consistent than verbal responses. Notably, motor response bias has been found to increase as a function of time since stimulus exposure and change in response context. This latter pattern is clearly illustrated in a set of studies by Creem and Proffitt (1998) involving memory-based motor and verbal estimates of the steepness of hills. Participants' motor estimates within a few seconds of viewing were very accurate, whereas verbal estimates from immediate memory were reliably too large. Given a delay of one day or when taken to a different location, participants' error in motor responses increased significantly, as did the overestimation evident in verbal judgments.

Creem and Proffitt (1998) interpreted such results as an evidence for two different memory systems. The first system involves storing information very briefly for a perceptually guided action. This system functions within an egocentric frame of reference and provides the information necessary for a rapid and precise responding within the task context. Information within this system decays quickly. The second system involves explicit memory, a cognitively penetrable product of effortful computation. This system produces a flexible representation that can be used within either an egocentric reference system to guide movement or an allocentric reference system to consider inter-object relations. Information processing within this system is posited as being comparatively more time consuming, more biased, and longer lasting, thus yielding enduring memory that is efficient but not necessarily precise. The two systems interact such that motor responses that are outside the temporal and spatial context in which the information was acquired are influenced by information represented in the explicit system, thus yielding bias (see also Neggers, Schölvinck, van der Lubbe, \& Postma, 2005; Parslow et al., 2005, for further insights in ego- and allocentric encoding of space). 
Clearly, one important challenge facing researchers is to arrive at a formal expression or model of how accuracy and bias are both incorporated in spatial memory. Such a model would be very useful in differentiating bias from error per se and in characterizing changes over time in the influence of multiple systems or processes of spatial memory. In this regard, the category-adjustment model of Huttenlocher et al. (1991) provides a firm conceptual foundation. In this model, every recollected spatial location is the product of two distinct coding processes, fine-grain and categorical. On one hand, fine-grain coding yields an unbiased representation of veridical location, with uncertainty reflected in a distribution of values around the true location. When recollected, the retrieved fine-grain value is a sample from that distribution (Huttenlocher et al., 1991). On the other hand, categorical coding yields a represented location based on a category prototype, typically the central location within an explicitly or implicitly bounded region. As with the fine-grain information, during retrieval a value is sampled from locations distributed around the prototype. According to the model, when an observer retrieves location information, faster decaying fine-grain memory and more robust categorical memory interact in a compensatory fashion. The predicted relationship is that the more uncertain the finegrain information, the greater the adjustment of estimated location consistent with categorical information.

This model provided an excellent description of performance in a spatial memory task in which observers reproduced the location of a dot presented briefly in a circular field by redrawing it into a circle on a sheet of paper in front of them (Huttenlocher et al., 1991). When considered within the context of the distinction between a perception-action system and an explicit memory system, performance of this task would be posited as involving principally the former. The momentary delay between presentation and response, the motoric nature of the response, and the constancy of the context would all lead to the prediction of high accuracy and negligible bias. But the predictions and findings of the study went substantially beyond the matter of more versus less bias. In fact, categorical bias was found as predicted by the model; in other words, the amount of bias depended upon observers' spontaneous tendency to impose categorical structure on the range of potential stimulus locations. In this specific task, that meant imposing implicit quadrants on the circular field (categories) and biasing memory-based responses toward the middle of those quadrants (prototypes) (see Huttenlocher et al., 1991). This finding has important implications for the tasks and outcomes used to support two-system accounts of the spatial memory.

Given this realization, we set out to investigate how the fundamental tenets of the category-adjustment model could be applied to a wider range of tasks. Specifically, we were interested in memory-based estimates of incline and azimuth. These phenomena were selected for several reasons. First, incline and azimuth are typical of spatial relations common in everyday life. We walk up and down naturally occurring slopes as well as ramps of various incline that are architectural features of the built environment (such as driveways and curb cuts); we use direction to target information to guide reaching, in navigation, and while communicating spatial information to others. Second, estimates of both incline and azimuth can be obtained through 
different response modes (verbal versus motor estimates, for example), thus providing an expanded range of outcomes to be predicted by the model. One of the most intriguing possibilities was that a single model involving the interplay of fine-grain and categorical spatial information could predict performance in not only different tasks (incline and azimuth estimation), but in different response modes (verbal and motor) within task. Third, both incline and azimuth estimations are directly analogous to the angular estimates of a target's radius within a circular field, data that were described very well by Huttenlocher et al.'s (1991) model. Fourth, bias has been documented previously in incline (Creem \& Proffitt, 1998) and azimuth (Montello, Richardson, Hegarty, \& Provenza, 1999) estimates. Fifth, estimates of incline and azimuth can be obtained by means of straightforward, readily replicable experimental procedures.

In Experiment 1, we adapted the category-adjustment model to apply to task settings involving an single implicit spatial category. The tasks under investigation included motoric and verbal estimates of incline and a azimuth. In Experiment 2, we expanded the investigation to involve a setting with multiple implicit categories, and we examined the impact of working memory load on bias in spatial memory. Azimuth estimation was selected as the task in that study. The purpose in both studies was to seek empirical evidence of the interplay of both fine-grain and categorical coding across all tasks and task settings.

\section{Adaptation of the category-adjustment model}

Because bias in estimation is central to our investigation, we present our formal model specifying its quantification in the single category case as follows. According to the category-adjustment model of Huttenlocher et al. (1991), the expected value $E$ of the estimate $R$ can be characterized as a weighted average of fine-grain and categorical information described by the following equation:

$$
E[R]=\lambda \mu+(1-\lambda) p,
$$

where $\mu$ is the true location of the object, $p$ is the expected prototype location, and $\lambda$ represents the relative weight of the fine-grain information. The random variable $M$ represents the memory recollection on any given trial and is assumed to be normally distributed about $\mu$, with standard deviation, $\sigma_{M}$, corresponding to the uncertainty of the information encoded into memory. Similarly, the random variable $P$ represents the category prototype location on a given trial, with mean, $p$, and standard deviation, $\sigma_{P}$, representing the uncertainty of recollection of the prototype. If only the fine-grain information were used, reports would be unbiased as $M$ is centered on the actual value, $\mu$. However, uncertainty about the fine-grain information leads to integration of the categorical information $(p)$ into the report, thus biasing estimates. Following Huttenlocher et al. (1991), we assumed that $\lambda$, or the weight of the fine-grain information should be close to 1.0 when uncertainty about fine-grain memory values is small and $\lambda$ should be close to 0 when uncertainty about fine-grain memory values is large. 
In psychophysical tasks, uncertainty is typically reduced at the endpoints of the range of values, as reflected in standard measures of discriminability, such as $d^{\prime}$ (Luce, Nosofsky, Green, \& Smith, 1982). The increase in uncertainty toward the middle of a linear series (or the corresponding increase in discriminability at the endpoints) is referred to as a "bowing effect". In the current application, the bowing effect argues stimulus discriminability, and hence weighting of fine-grain memory $(\lambda)$, should be maximal at the endpoints of the natural category (i.e. $0^{\circ}$ and $90^{\circ}$ ). We used a simple quadratic function to approximate this relationship, predicting fine-grain memory weight as a function of the distance between true angle and prototype:

$$
\lambda=a+b(\mu-p)^{2}
$$

where $a$ and $b$ are constants, representing the general weighting of fine-grain information, and how the weighting of fine-grain memory information changes as a function of distance from the prototype, respectively.

In the category-adjustment model, the focus is on bias, or how estimates deviate from actual values. Substituting the expression for $\lambda$ from Eq. (2) into Eq. (1) and solving for bias yields the following equation that we use to fit the data:

$$
\operatorname{Bias}(R)=(\mu-p)\left(a+b(\mu-p)^{2}-1\right) .
$$

Altogether, three values are free to vary in Eq. (3): $p$, representing the location of the prototype, $a$ and $b$.

Fig. 1 illustrates the model's prediction of bias under the condition of constant weighting, when $b=0$ (dashed line), and under the condition of a hypothesized curvilinear relationship between discriminability and target value, when $b>0$ (solid line). Panel $\mathrm{C}$ plots these relationships assuming that the prototype lies near midrange (i.e., at $45^{\circ}$ ). Panel A shows the situation when $\lambda$ equals a constant, and panel $\mathrm{B}$ shows the condition of a curvilinear relationship between target value and $\lambda$. The prototype model predicts a straight-line function when $\lambda$ equals a constant, with the greatest bias at the endpoints of the range and zero bias at the prototype. The curvilinear relationship between $\lambda$ and target value portrayed in panel $\mathrm{B}$ produces a cubic trend in which bias decreases near the endpoints, as greater discriminability leads to greater weighting of the fine-grain representation.

\section{Experiment 1}

The purpose of this study was to examine the fit of our modified category-adjustment model to data from single-category versions of incline and azimuth estimation tasks. As mentioned previously, bias has been found in these phenomena (Creem \& Proffitt, 1998; Montello et al., 1999). However, fitting data from both tasks to a single model is a unique and a theoretically significant undertaking.

The inclusion of both motoric and verbal modes of estimation in this study was motivated by the intriguing finding of Creem and Proffitt (1998) that immediate memory-based verbal estimates of incline were subjected to substantial bias while 


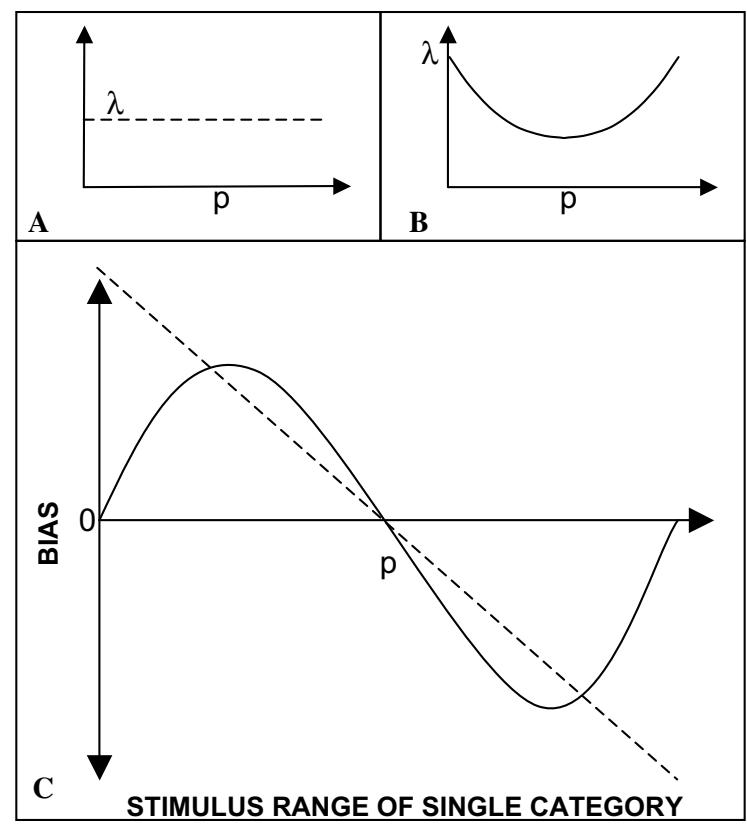

Fig. 1. Model prediction of bias under conditions of constant weighting of fine-grained information $(b=0)$ and under conditions of a curvilinear relationship between weight and angle $(b>0)$. Panel A: Condition of $\lambda$ equaling a constant. Panel B: Condition of a curvilinear relationship between target value and $\lambda$. Panel C: Resulting relationships assuming that the prototype lies near the middle of the range. Solid line corresponds to curvilinear weight and dashed line to constant weight.

motoric estimates were not. Furthermore, they argue that the bias in verbal estimates reflected a consistent tendency to overestimate incline. The task in their study was to estimate the geographic slant of hills in natural settings, which is substantially different from estimating the incline of small ramps in the laboratory. Thus, ironically, we were curious to determine whether the findings from the field would generalize to the laboratory. In that regard, we noted that the inclines in Creem and Proffitt's (1998) study were naturally restricted to a relatively narrow range. This is an important point in the context of the category-adjustment model, which would predict overestimation of values smaller than the category prototype and underestimation of values larger than that prototype. Accordingly, we designed our study with an expanded range of inclines to be estimated so that values throughout the hypothesized spatial category $\left(0-90^{\circ}\right)$ were included.

We hypothesized that verbal estimates of incline and azimuth would include substantial categorical bias and would be accurately fit using the modified categoryadjustment model. Based on Creem and Proffitt's (1998) report of undistorted estimates of incline using a tilt board, we were more cautious regarding motoric estimates. Nonetheless, we expected that if bias were detected in motoric estimates, it would fit the pattern prescribed by our model. 


\subsection{Method}

\subsubsection{Participants}

Fifty-two undergraduate students (26 male and 26 female), ranging in age from 18 to 25 years $(M=19.9 ; \mathrm{SD}=1.5)$, participated in the experiment for class credit. All participants were naïve to the purpose of the study and were free of visual or motor problems that would have influenced their performance in the tasks.

\subsubsection{Task and materials}

Targets for the incline estimation task were created by leaning a black 80 by $100 \mathrm{~cm}$ Styrofoam board, with a white grid drawn on it, against a black wall. The angle between the board and the floor was defined to be $4^{\circ}, 24^{\circ}, 43^{\circ}, 68^{\circ}$, or $86^{\circ}$. Participants were positioned to face the incline from a distance of approximately $1.5 \mathrm{~m}$. A tilt board mounted on a tripod, approximately $1 \mathrm{~m}$ in height was used for motor estimates. Verbal estimates were given in degrees, with $0^{\circ}$ representing ground level. Participants made all estimates while blindfolded with opaque goggles (see Fig. 2A).

The target stimulus for the azimuth estimation task was the $2 \mathrm{~cm}$ white tip of a black, wooden dowel that was $50 \mathrm{~cm}$ in total length and $1.2 \mathrm{~cm}$ in diameter. The dowel was mounted vertically on a black tripod so that the target height was approximately $1 \mathrm{~m}$. Participants stood $1.5 \mathrm{~m}$ from a black curtain mounted from ceiling to floor that formed roughly a quarter-circle $\left(105^{\circ}\right)$ in front of and to the left observer. Participants were told that straight ahead was a value of $0^{\circ}$. The five specific stimuli to be estimated were $2^{\circ}, 24^{\circ}, 49^{\circ}, 71^{\circ}$, and $88^{\circ}$ offset to the left. Motor estimates were made with a pointing device involving another dowel, $85 \mathrm{~cm}$ in length and $1.2 \mathrm{~cm}$ in diameter, mounted horizontally on a second tripod at $1 \mathrm{~m}$ height. A protractor was mounted on the supporting tripod to facilitate the response measurement. Thus, participants produced motor estimates by aiming the pointing device and verbal estimates by judging number of degrees to the left of straight forward. Participants wore opaque goggles while making their estimates (see Fig. 2B).

\subsubsection{Procedure}

Participants were tested individually in a large black room. First, the experimenter guided each participant to the observation position in front of the tilt board device. A trial began by asking the participant to look at an incline in order to memorize it. The angle could be viewed as long as deemed necessary by the participant. The individual then placed the opaque goggles resting on their forehead down over their eyes. The participants blindfolding themselves took approximately $3 \mathrm{~s}$. Then they were asked to give a verbal estimate in degrees and a motor estimate by mimicking the memorized incline by setting the tilt board to the same angle. This procedure was repeated for four more angles adding up to a total of five verbal and five motor estimates for every individual. Half the participants were randomly assigned to make verbal estimates first on each trial, with the other half making motor estimates first. Order of presentation of the five target angles was randomized for each individual. After estimating the last slant, participants were guided to a second observation 


\section{Incline-Task Exp 1}

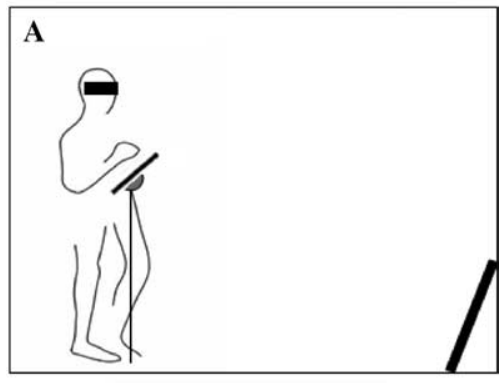

Azimuth-Task Exp 1

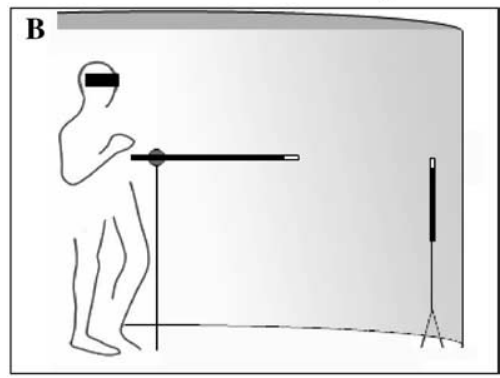

Azimuth-Task Exp 2

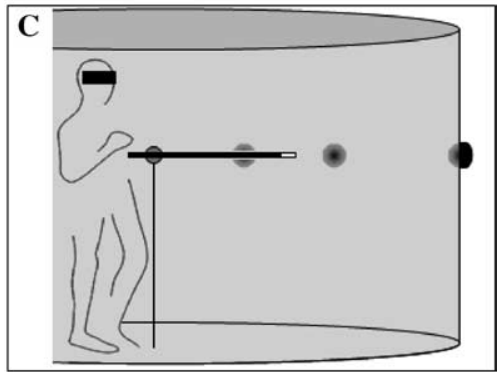

Fig. 2. Schematic drawings of the experimental setups in Experiment 1 for incline (A) and azimuth estimates (B) and the azimuth estimates in Experiment 2 (C).

position for azimuth estimations. The azimuth estimation task was administered in a way comparable to the slant estimation task.

\subsection{Results}

Bias scores were computed for each participant and submitted to one-way, repeated-measures analyses of variance (ANOVAs) conducted separately for verbal and motor estimates. We predicted bias to be revealed as cubic or linear trends (see Fig. 1). Fig. 3 presents the bias scores along with model estimates. 


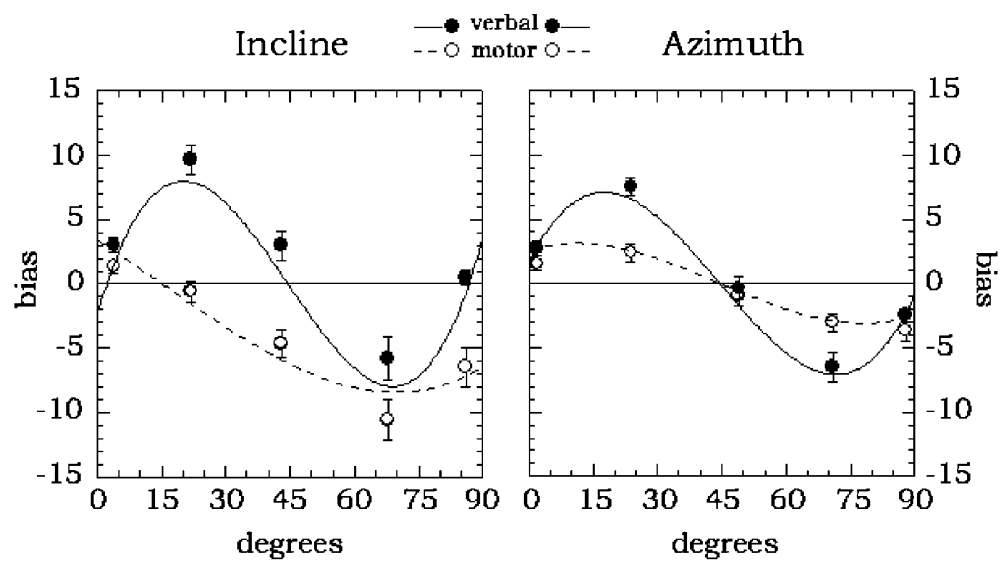

Fig. 3. Mean signed bias scores in verbal and motor estimates of incline and azimuth. The error bars indicate the standard error of the mean. Functions indicate model fit to empirical means of bias scores in verbal and motor estimates of incline and azimuth (see Eq. (3)).

Consistent with the predicted indicator of bias, a significant cubic trend was revealed for bias scores in verbal estimates of incline, $F(1,51)=50.06, p<.0001$, $\eta^{2}=.495$, and verbal estimates of azimuth, $F(1,51)=61.24, p<.0001, \eta^{2}=.546$. Also a significant linear trend was detected for bias scores in verbal estimates of incline, $F(1,51)=62.0, p<.0001, \eta^{2}=.549$ as well as for bias scores in verbal estimates of azimuth, $F(1,51)=137.43, p<.0001, \eta^{2}=.729$. As shown in Fig. 3, bias for verbal estimates was minimal near the endpoints of the range $\left(0^{\circ}\right.$ and $\left.90^{\circ}\right)$, as well as for the midpoint of the range that should correspond to the category prototype (i.e., $45^{\circ}$ ).

Surprisingly, in the light of prior research showing a lack of bias for motor estimates, the ANOVAs conducted on motor bias scores of incline and azimuth also revealed significant cubic trends for incline, $F(1,51)=12.80, p=.001, \eta^{2}=.201$, and for azimuth, $F(1,51)=11.46, p=.001, \eta^{2}=.183$. Significant linear trends were also detected for motor estimates of both incline and azimuth, $F(1,51)=42.99, p=.0001$, $\eta^{2}=.457$ and $F(1,51)=46.63, p=.0001, \eta^{2}=.478$, respectively.

The mean bias scores were fit to the model of Eq. (3) using the iterative nonlinear regression procedure within SYSTAT (Wilkinson, 1989), with a least squares error criterion and the Gauss-Newton method of steepest descent. The set of 20 target means was fit simultaneously, with 12 parameters free to vary. Parameters were constrained as long as the result was not a significant drop in $R^{2}$. The final model had 10 free parameters, with $a$ and $b$ fit to each condition separately, a single prototype value fit to three of the conditions, and a separate prototype value fit to the motor incline estimation data. Table 1 presents the estimated parameter values, and Fig. 3 the fit of the model to the data. The model provided a good account of bias in the two estimation tasks under the two response modes, with variance accounted for being reasonably high, $R^{2}=0.939$. 
Table 1

Parameter values for model fit

\begin{tabular}{lllll}
\hline Task & Mode & Parameter & & \\
\cline { 3 - 5 } & & $P$ & $a$ & $b$ \\
\hline Incline & Motor & $15.11^{\circ}$ & 0.768 & .000026 \\
Incline & Verbal & $44.53^{\circ}$ & 0.511 & .000274 \\
Azimuth & Motor & “ & 0.860 & .000042 \\
Azimuth & Verbal & “ & 0.607 & .000180 \\
\hline
\end{tabular}

Note: " indicates parameter value constrained to be equal to value above; $p=$ prototype value, $a=$ additive constant defining $\lambda ; b=$ weight of quadratic component defining $\lambda$.

Model parameters confirmed the ANOVA results. The higher values for motor than for verbal estimates correspond to generally greater weighting of fine-grain information $(\lambda)$ for the former. Also, verbal estimates showed a greater reduction in bias at the extreme target values than did motor estimates, as reflected in higher values of $b$ (reflecting the bowing effect for $\lambda$ ).

\subsection{Discussion}

Overall, our model fitting effort showed that estimates in both the verbal and motor versions of both incline and azimuth estimation tasks could adequately be described by our adaptation of Huttenlocher et al.'s (1991) category-adjustment model, in which estimates are a product of both fine-grain and categorical memory coding. We had hypothesized that this would be the case for verbal estimates unequivocally and for motoric estimates to the extent that bias was detectable.

Embedded within our results for incline estimation was a replication of the previous finding that, for angles smaller than $45^{\circ}$, motor estimates of incline were quite accurate and verbal estimates were systematically too large. Thus, a fundamental distinction made by Creem and Proffitt (1998) was replicated in the lab with small-scale inclines, although the magnitude of verbal overestimation was not as great as with hills. Nonetheless, this pattern embedded in our data was shown to be part of a larger story. The overall profile of motor estimates to targets over a $90^{\circ}$ range reflected not only considerable accuracy but also significant bias towards overestimating smaller angles and underestimating larger ones. This same signature bias was dominant in the verbal estimation data.

Categorical bias in motor estimates and the verbal underestimation of inclines greater than $45^{\circ}$ are in contrast to predictions from Creem and Proffitt (1998). However, categorical bias in motor estimates is compatible with the idea of constantly interactive perception-action and cognitive systems as described in recent papers by Creem and Proffitt (2001a, 2001b), Diedrichsen et al. (2004), and Werner and Diedrichsen (2002). With regard to verbal underestimation, it must be acknowledged that small inclines in the laboratory are not the same as hills in natural settings. Indeed, consistent with Creem and Proffitt's prediction, observers of steep hills in virtual environments tend to overestimate those inclines (Proffitt, Bhalla, Gossweiler, \& 
Midgett, 1995). Clearly, the affordances of inclines have implications on estimation (Bhalla \& Proffitt, 1999; Proffitt, Stefanucci, Banton, \& Epstein, 2003). Nevertheless, small-scale inclines are common features in natural and built environments, and the lack of generalizability between research findings for hills and those for smaller inclines is noteworthy.

The findings of this experiment set the stage for expanding the modified categoryadjustment model to a task with more than one implicit category, in other words, to a range of potential responses that include more than $90^{\circ}$. Inspection of the functions in Fig. 3 suggested azimuth estimation to be a useful task for this purpose. The data showed a prototypic value at the center of the implicit category for both verbal and motoric modes of estimation for azimuth. This was not the case for incline estimation, probably because of the restricted range of wrist motion available for motoric estimates on a waist-high palm board. No such restriction applied to motor estimates of azimuth. Also, the data for estimates of azimuth provided an excellent fit to the model. For these reasons, motoric estimation of azimuth was selected as the task for Experiment 2.

\section{Experiment 2}

The purpose of Experiment 2 was to test two hypotheses regarding categorical coding of spatial relations. The first hypothesis was that our modified categoryadjustment model developed in Experiment 1 could be adapted for application to a multiple category task setting by specifying an additional process. Our adaptation is based on the uncertain boundaries version of the category-adjustment model described by Huttenlocher et al. (1991), which posits that near the borders between categories there will be times when the same stimulus elicits different category prototypes (due to unbiased error distributions posited for stimulus and category prototype locations). Following this approach, the observer makes an initial determination regarding the category in which a target is included. One possibility is that the observer perfectly discriminates central angles so that the prototype from the correct quadrant is always retrieved and integrated into judgment. In such cases, the model of Eq. (3) could simply be fit directly to the data, with the right prototype always retrieved when the angle was from the right quadrant and the left prototype always retrieved when the angle was from the left quadrant. However, Experiment 2 was designed to tax observer's memory for the stimuli and thus it seemed unlikely that the correct prototype would be reliably retrieved in all cases. We therefore extended our modified model to include an additional parameter that indicated the probability of retrieving the right and left prototypes given the presented angle. We chose a logistic function to model this fuzzy boundary because the function is both mathematically simple and psychologically plausible (see Link, 1992). Thus, the probability of retrieving the prototype from the right quadrant $\left(p_{R}\right)$ given a particular angle $X$ was determined as follows:

$$
\operatorname{Pr}\left(p_{R} \mid X\right)=1 /(1+\exp (-c X)),
$$


where negative values corresponding to angles from the left quadrant and $c$ is a discriminability parameter. Eqs. (3) and (4) can then be combined to model the two category situation as follows:

$$
\begin{aligned}
\operatorname{Bias}(R)= & \left(1-\operatorname{Pr}\left(p_{R} \mid X\right)\right)\left(\mu-p_{L}\right)\left(a+b\left(\mu-p_{L}\right)^{2}-1\right) \\
& +\operatorname{Pr}\left(p_{R} \mid X\right)\left(\mu-p_{R}\right)\left(a+b\left(\mu-p_{R}\right)^{2}-1\right) .
\end{aligned}
$$

Eq. (5) describes the pattern of bias in terms of five fitted parameters, the two inferred prototypes $\left(p_{L}\right.$ and $\left.p_{R}\right)$, the weighting of fine grained information, $a$, the tendency to show the bowing effect, $b$, and the sharpness or discriminability of the boundary assumed to be located at angle $0^{\circ}$, and the discriminability parameter $c$.

Note that the core elements of the category-adjustment model remain unchanged in this version of the model, namely that two independent memory representations, fine-grained and categorical, are combined in the estimation process. What Eqs. (4) and (5) introduce is uncertainty with regard to categorical classification of a stimulus modifies the bias effects occurring near category boundaries, referred to as the uncertain boundaries model by Huttenlocher et al. (1991).

In addition to this hypothesis concerning the adaptation of the model, our second hypothesis in Experiment 2 was that the observer's reliance on categorical coding should be affected by factors known to decrease the precision of memory, namely memory load, delay, and disruption. Specifically, we expected that adding more factors that produce uncertainty would produce a graded increase in reliance on categorical coding. Accordingly, we expected that holding three locations in working memory while awaiting indication of which one is the target would result in an increased tradeoff of lower effort categorical coding for higher effort fine-grained coding (Baddeley, 1986; Logie, 1995). We further expected that delay in responding would produce increased evidence of categorical coding compared to immediate responding based on the finding of time-sensitive decay from visual-spatial working memory (Baddeley, 1986; Logie, 1995), in combination with the assumption that fine-grain and categorical information decay at different rates (Creem \& Proffitt, 1998; Huttenlocher et al., 1991). Finally, we expected that disruption in the form of an intervening visual-motor task would tax visual-spatial working memory (Logie, 1995) and thus result in pronounced reliance on categorical coding compared to performance when no disruption task was present. The expectation was based on the assumption that categorical coding is maintained in memory with less effort than is fine-grain information (Huttenlocher et al., 1991).

\subsection{Method}

\subsubsection{Participants}

Twelve male and 18 female undergraduates, with an age-range of 17 to 33 years $(M=20.14 ; \mathrm{SD}=3.18)$ of the University of South Carolina participated in the reported experiment in return for class credit. All participants were naïve to the purpose of the study, had no knowledge of American Sign Language, and had no visual or locomotor problems that would influence performance in the experimental tests. 


\subsubsection{Task and materials}

The setting for the azimuth estimation task was a $210^{\circ}$ semicircular area approximately $2.5 \mathrm{~m}$ in diameter formed by suspending a thin black curtain from ceiling to floor in the laboratory. Participants in the procedure stood in the center of the semicircle facing the midpoint of the curtain. An array of nine colored lights $1 \mathrm{~m}$ above the floor was mounted on the reverse side of the curtain so each presented a $10 \mathrm{~cm}$ color target when illuminated. The array consisted of a blue light at $96^{\circ}$ left of midpoint, a red light $77^{\circ}$ left of midpoint, a red light $52^{\circ}$ left of midpoint, a violet light $30^{\circ}$ left of midpoint, a yellow light $7^{\circ}$ left of midpoint, a blue light $22^{\circ}$ right of midpoint, a blue light $46^{\circ}$ right of midpoint, a yellow light at $72^{\circ}$ right of midpoint, and a violet light at $91^{\circ}$ right of midpoint. These angles were selected to cover two spatially contiguous categories of $90^{\circ}$ each. The lights were controlled by a series of switches under the control of the experimenter, who sat behind the participant during the procedure. Azimuth estimates were made using the pointing device described in Experiment 1. During all estimates, participants wore opaque goggles (see Fig. 2C).

Trials in the azimuth estimation task involved presentation of either one target (lower memory load) or three potential targets (higher memory load). With the higher memory load, the target was identified after the stimuli were no longer visible. The task also involved three conditions involving potential disruption of memory: immediate estimation (no disruption), estimation after $15 \mathrm{~s}$ delay (no disruption with delay), and estimation after a disruption task (disruption with delay). The disruption task involved copying a videotaped presentation of a young woman spelling common words using American Sign Language (ASL-task). This task was selected because it involved movement as well as visual memory and, thus, could be expected to disrupt all hypothesized relevant components of visual-spatial working memory (Logie, 1995). Signing was continuous but at a rate slow enough to be imitated by naïve observers. The presentation was shown to participants on a video screen mounted so that it faced upwards right above floor level. The screen was immediately in front of the participants' viewing position. During testing, the disruption task was presented for $10 \mathrm{~s}$ prior to when the participants were blindfolded and made their responses to the azimuth estimation trial. The time elapsed from viewing the target(s) to estimating their azimuth was approximately $15 \mathrm{~s}$, which matched the interval in the condition that included a delay with no disruption.

\subsubsection{Procedure}

Participants were tested individually in a large room. Initially, the azimuth estimation task was explained to them and they familiarized themselves with the mounted dowel used to respond in the task. The disruption task was also explained and demonstrated. After the instructional phase of the procedure, each participant responded in 54 trials. Half of the trials involved presentation of a single target, and the other half involved presentation of three potential targets. One-third of the trials involved responding immediately (short delay condition), one-third involved responding after a $15 \mathrm{~s}$ delay with no disruption task (long delay condition), and one-third involved responding after performing the disruption task (ASL condition). These conditions were blocked and their order was counterbalanced. On any given trial stimuli were 
presented for $1 \mathrm{~s}$. Participants were encouraged to look at the presented stimuli in order to memorize them. The individual then placed the opaque goggles resting on their forehead down over their eyes, introducing a minimal delay of approximately $3 \mathrm{~s}$ between presentation and response (short delay), which was extended in the long delay and ASL conditions. After the delay period, they were asked to produce an estimate using the pointing device. Order of presentation of the target angles was randomized for each individual.

\subsection{Results}

A one-way repeated measures ANOVA was performed on the signed angular deviation of estimates to examine the influence of stimulus location within spatial categories on the response bias. An a priori hypothesis predicted quintic trends in all six conditions, increasing in strength with increase in load and disruption. Assuming equally spaced stimuli the analysis revealed significant quintic trends in all conditions. Compared with other polynomial trends up to the seventh order, the quintic contrast accounted for the largest effect sizes. $\left(F(1,29)=14.63, p=.001, \eta^{2}=.335\right.$ for low load short delay; $F(1,29)=19.20, p=.0001, \eta^{2}=.398$ for low load long delay; $F(1,29)=10.55, \quad p=.003, \eta^{2}=.267$ for low load ASL-filled delay; $F(1,29)=16.503, p<.001, \eta^{2}=.363$ for high load short delay; $F(1,29)=13.05$, $p=.001, \eta^{2}=.310$ for high load long delay; and $F(1,29)=19.15, p=.0001$, $\eta^{2}=.398$ for high load ASL-filled delay).

A 2 (memory load) by 3 (delay conditions) repeated measures ANOVA was performed on the quintic contrast scores generated for every individual in each condition. These contrast scores represent the strength of categorical influences on that individual's estimates. A Greenhouse-Geisser correction adjusted for violations of sphericity where appropriate. The analysis revealed no significant main effect of memory load $(F(1,29)=2.89, p=.1)$ or delay $(F(2,58)=.42, p=.611)$. After correcting for multiple comparisons no simple contrasts between any of the conditions were significant.

The mean bias scores were fit to our modified category-adjustment model as adapted to the multiple category setting using the iterative nonlinear regression procedure within SYSTAT (Wilkinson, 1989), with a least squares error criterion and the Gauss-Newton method of steepest descent to fit Eq. (5) to the data. The least constrained version of the model allowed each of the five parameters of Eq. (5) to vary freely across experimental conditions and resulted in a total of 30 parameters to fit 54 data points with $R^{2}=0.818$. The most constrained version of the model held the values of the five parameters constant across experimental conditions and resulted in a total of five parameters to fit 54 data points with $R^{2}=0.667$. We took this five-parameter base model and successively freed parameters that led to a significant increment in $R^{2}$. Our final model used nine free parameters to fit the 54 data points with $R^{2}=0.782$. The fit of this model is shown in Fig. 4.

As displayed in Fig. 4, the data show the characteristic quintic pattern predicted by the model. Bias is minimized near the middle of each category and at the boundary of categories. Furthermore, bias is positive for angles lower than the correspond- 


\section{Degrees Azimuth}
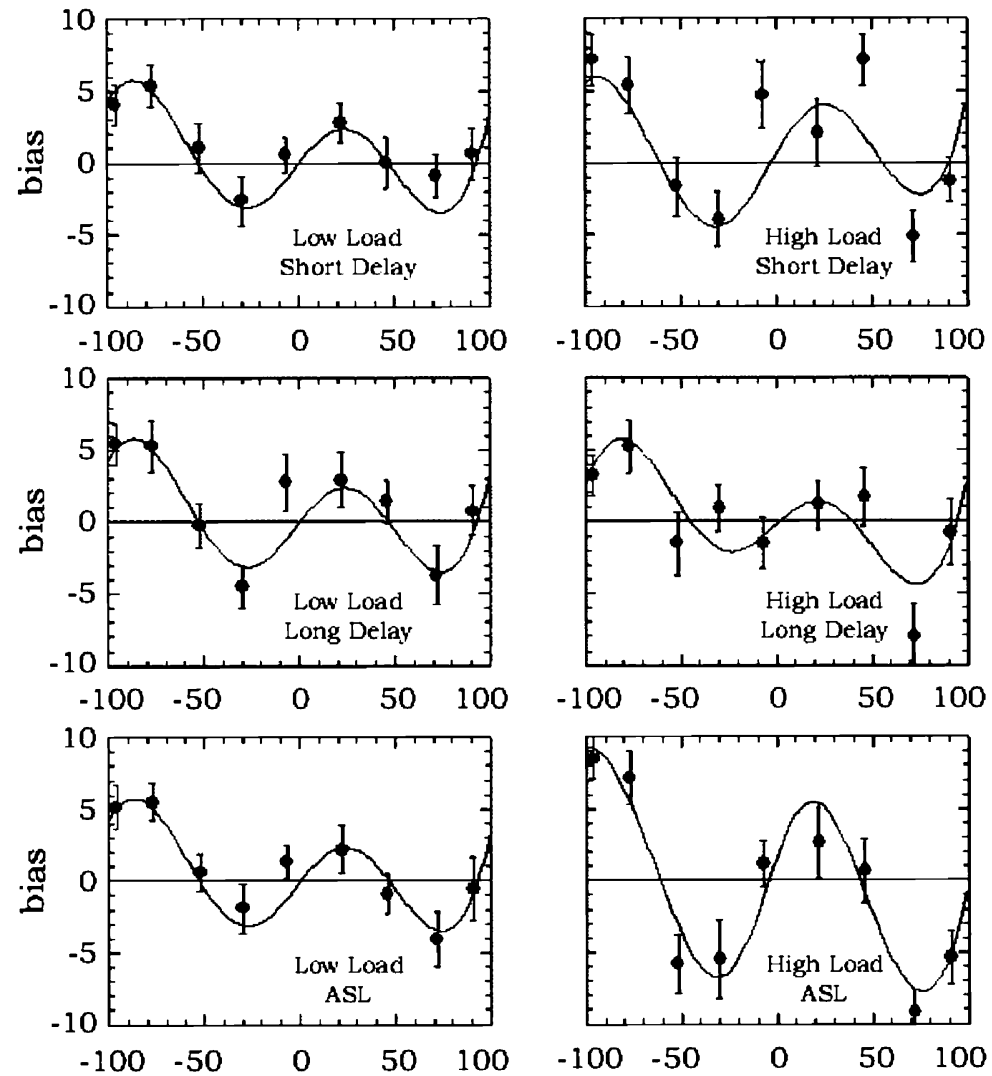

Fig. 4. Mean signed bias scores in motor estimates of azimuth (Experiment 2). The error bars indicate the standard error of the mean. Functions indicate model fit to empirical means of bias scores under the six experimental conditions (see Eq. (5)).

ing prototype, but is negative for angles higher than the corresponding prototype. Although the same general pattern is evident in all six conditions, the high load conditions (in which multiple potential targets were presented) show a less orderly pattern.

The same five parameter values were held constant across the low load conditions. The inferred weighting of fine-grain memory $(a=0.63)$ was reduced relative to its inferred value for the motor estimation of azimuth in Experiment 1 and indeed was close to the value inferred for verbal estimates in that task. This may be attributed to the well-known reduction of stimulus discriminability with increase in stimulus range (Gravetter \& Lockhead, 1973). The non-zero value of the bowing parameter $(b=.000078)$ reflected the tendency to show less bias at the borders of the category, consistent with increased discriminability for stimuli near the border. The inferred value for the left hemisphere prototype $\left(P_{L}=-45.78\right)$ was very close to that found 
for Experiment 1 and represented approximately the center of that $90^{\circ}$ quadrant. The inferred value for the right prototype $\left(P_{R}=32.80\right)$ was not symmetrically located at the center of the $90^{\circ}$ quadrant but rather was shifted closer to $0^{\circ}$. Finally, the low value of the boundary parameter $(c=.036)$ reflected a fair amount of confusion in selecting the appropriate prototype for the target angle (such that a stimulus at angle $30^{\circ}$ had about a one fourth probability of recruiting the wrong prototype).

The fit to the high load conditions used the same constant parameter values for the memory parameter $(a)$, the bowing parameter $(b)$, and the prototype for the right hemisphere $\left(P_{R}\right)$ as were inferred for the low load conditions. To fit differences across these conditions, the prototype for the left hemisphere was free to vary, with values of $\left(P_{L}=-52.09\right.$ for short delay, $P_{L}=-40.91$ for long delay, and $P_{L}=-58.55$ for the ASL delay task). The ASL delay task also required a slightly higher value of the boundary parameter $(c=.056)$. These differing parameter values seem somewhat idiosyncratic and may simply reflect the greater confusion participants had when multiple potential targets were presented.

\subsection{Discussion}

The purpose of Experiment 2 was to develop a multiple-category version of our adaptation of Huttenlocher et al.'s (1991) category-adjustment model and determine the ability of that model to fit data from a task in which participants made memory-based azimuth estimates. First, it should be noted that, consistent with results from Experiment 1 and previous research (e.g., Montello et al., 1999), our data revealed bias in memory-based motoric estimates of azimuth. Furthermore, the basic bias pattern for the two-category task setting, which was reflected in the condition in which there was a single target, short delay, and no intervening task, was very well predicted by our model (see the upper left-hand panel in Fig. 4). Of course, our primary focus of interest was the overall fit of the model across the six conditions. In this regard, the model can be described as providing a good account of the data.

It had been predicted that the various combinations of memory load, delay, and intervening task would result in a graded effect in terms of the magnitude of the quintic component of the response function. This effect was not observed. The main parameter within our model that corresponded to fine-grain information (the a parameter) was lower in Experiment 2 than in Experiment 1, most likely due to the increased range of values. Unexpectedly, letting this parameter vary with load or delay did not improve the fit of the model. With little previous research to guide generalizations about the sensitivity of fine-grain and categorical information to cognitive load or delay, we are left to speculate that in simple experimental settings such as ours fine-grain information degrades rapidly and then plateaus to a situational maximum. A rapid increase in categorical influence on coding, which is compatible with this view, has been documented in the previous research (Bridgeman, Peery, \& Anand, 1997; Diedrichsen et al., 2004; Werner \& Diedrichsen, 2002). 
The same factors that might be responsible for obscuring the anticipated graded effect may have also resulted in a fit to the data that was good rather than excellent. One probable source of this noise in the data was interference involving color-location associations, both within trials in the condition involving multiple potential targets and between trials across the procedure. Remembering where a target appeared is different psychologically from remembering which target appeared where (see Postma, Kessels, \& van Asselen, 2004).

\section{General discussion}

The biased yet accurate nature of spatial memory poses an interesting paradox for researchers studying how people acquire and make use of information about spatial relations. It is clear that a comprehensive account of spatial memory will have to include consideration of factors responsible for both geometric fidelity and bias. Accordingly, we focused our efforts on the category-adjustment model of Huttenlocher et al. (1991), which provides a formal statement of the interplay between fine-grain and categorical coding of spatial relations. Although the model was originally developed to account for location memory within a two-dimensional circular field external to the viewer, we hypothesized that with modifications it could apply equally well to a range of spatial phenomena, including memory for incline and azimuth from the viewer's perspective.

The single-category version of this model provided an excellent fit to the data from Experiment 1, and the multiple-category version provided a good fit to the data from Experiment 2. Regardless of what other factors may influence spatial memory in these tasks, the evidence seems consistent with the major influences being coding of fine-grain location information and categorical information based on geometrically determined prototypes.

Although we selected the category-adjustment model for our investigation, there are other models that can be used to describe the effects observed in our data. For example, Hollands and Dyre's (2000) cyclical power model predicts a pattern of results that strongly resembles those predicted by the category-adjustment model. ${ }^{1}$ Our study was not designed as a comparison between these models and thus, we

\footnotetext{
${ }^{1}$ According to the cyclical power model, bias results from comparison of values resulting from a power transformation of the physical scale in accordance with Steven's power law (Hollands \& Dyre, 2000). Power coefficients less than one result in the pattern of bias observed by Huttenlocher et al. (1991) and power coefficients greater than one result in the opposite pattern. The degree of bias decreases as the power exponent approaches one. The cyclical bias occurs within a segmented range of values defined by a minimum and maximum value. Multiple cycles occur when there are multiple subranges. We fit basic versions of the cyclical power model to the data of Experiments 1 and 2. The cyclical power model provided a comparable fit to the data of Experiment 1 with a similar number of fitted parameters, but it provided a slightly poorer fit to the data of Experiment 2 with more fitted parameters than we used. Altogether, the fits of the models do not provide a very strong rationale for one model over the other. Thus, the selection of models rests on theoretical grounds.
} 
do not provide a detailed presentation of the cyclical power model's assumptions and fit. However, we emphasize that our choice of model was driven by theoretical concerns, namely, (a) the identification of a model that deal explicitly with memory and the factors responsible for recall and (b) a model that posits dual processes in the service of memory.

A number of different findings from research in spatial perception and cognition have given rise to two-system accounts of spatial memory. In the two-system account, a perception-action system, posited as functioning without awareness to provide information for immediate, high-precision responses, is distinguished from a cognitive system, posited as functioning within awareness to support a more enduring, multi-purpose representation that is biased but constrained in terms of error (Bridgeman et al., 1997; Creem \& Proffitt, 1998, 2001a; Goodale \& Milner, 1992). These systems are considered to be neurophysiologically distinct but functionally interactive (Creem \& Proffitt, 1998, 2001a, 2001b; Milner \& Goodale, 1995; Rossetti, 1998).

One approach to differentiating between the results of the two systems involved contrasting memory-based verbal and motoric estimates of spatial relations. Creem and Proffitt's (1998) studies of incline estimation provide a case in point. These investigators found that verbal estimation of a hill's incline resulted in estimates that were consistently too large, whereas motoric estimates were accurate and unbiased (Creem \& Proffitt, 1998). This pattern is consistent with a distinction between a cognitive system that yields bias and a perception-action system that is minimally biased, at least initially. In conducting Experiment 1, we were aware that replication and extension of the previous findings of biasfree motor estimates and biased verbal estimates would provide corroborating evidence for the two-system approach. In contrast to this pattern, however, our results showed similar bias across response modes and tasks. This outcome raises the possibility that the expanded range of target values in our study afforded a somewhat broader picture of both motor and verbal estimates in which categorical bias could be detected. If this possibility is true, then the pattern observed by Creem and Proffitt (1998) could be considered embedded in this broader picture. However, because of important tasks differences - Creem and Proffitt (1998) participants were estimating the slant of hills in the natural environment and ours were estimating small-scale inclines in the laboratory-it is not the case that one set of findings invalidates the other. Nevertheless, the incompatibility of outcomes does complicate the theoretical picture somewhat and suggests a need for future efforts at clarification.

Another question raised by our findings has to do with the relation between our results involving memory for azimuth in Experiment 2 and the results from Montello et al. (1999), which also showed evidence of bias. Although both sets of results show bias, bias-signatures appear to be different. Using the concepts from the categoryadjustment model, it appears that either the implicit categories or the prototypes within that categorical structure differ between the two studies. Without experimental work focusing directly on this issue, the factors contributing to the differences cannot be elucidated. 
Nevertheless, this consideration raises the general issue of bias within different spatial frames of reference. It is reasonable to posit that memory-based motor estimates of incline and azimuth in these studies reflected bias within an egocentric reference system, that is, a system in which the axes of the observer's body serve as a means of remembering the location of the target objects. Actually, the verbal estimates from a fixed observer viewpoint in these tasks involve the same body-based axes. However, as recent findings have made clear, features within the environment can exert a significant influence on how spatial relations are represented in memory, inducing the use of an allocentric frame of reference (Burgess, Spiers, \& Paleologou, in press; Diedrichsen et al., 2004; Mou \& McNamara, 2002; McNamara, Rump, \& Werner, 2003). The role of the response fields in these studies (for example, the floor and the wall for the inclines and the semicircular fields for the azimuths) afforded observers the opportunity for allocentric coding of spatial relations. In our procedure, we cannot differentiate between the influences of imposing an implicit categorical scheme based on body axes and those of imposing an implicit categorical scheme on the response field. Clearly, differentiation between these influences would be an interesting and informative agenda for future studies.

An additional issue of significance that arises in light of our results is the time course of bias in verbal and motor estimates of spatial relations. Recent experiments have demonstrated that motor response bias in a memory for location task can appear within $50 \mathrm{~ms}$ of initial exposure to stimulus location (Diedrichsen et al., 2004; Werner \& Diedrichsen, 2002). Our findings of categorical motor response bias in incline and azimuth estimates made within a few seconds of viewing the stimulus are consistent with these recent data. Nevertheless, our motivation for these studies was specifically to examine the theoretically based and empirically supported prospect that memory-based motor estimates would be unbiased, not just for a few seconds but for substantially longer periods of time (see Creem \& Proffitt, 1998). Although our data say little about the time course of such bias, they clearly support the position that it appears early and is robust. Also, because we saw no significant increase resulting from delay, memory load, or disruption in Experiment 2, our data also suggest the possibility that motor bias per se may be constrained under taskdependent maxima.

Cognizant of unresolved issues and of the constraints of our data, we nevertheless categorically endorse the idea that categorical bias can exert a significant influence on both memory-based motor and verbal estimates of spatial relations, and we further suggest that results such as these have significant implications for two-system theories of spatial memory. If distinct systems are posited, then they must be interactive as well as complementary. Consequently, the issue of how to distinguish between the systems on the basis of behavioral criteria requires additional consideration. Differences in time necessary for initial coding, robustness over retention intervals, and sensitivity to contextual factors present during coding are factors that may show promise in this regard.

Despite empirical and theoretical progress in spatial memory research, many important issues have yet to be resolved. It is clear that in human spatial memory, information can be represented at both a fine-grain and a categorical level. It is 
not so clear, whether we should think of this information as the product of separate perception-action and cognitive systems. Evidence suggests that fine-grain information decays rapidly, but as of yet it is difficult to generalize rates and limits of decay across tasks and situations. It is safe to say that categorical coding exerts a significant influence on how we conceive of spatial relations in a variety of contexts, but much remains to be learned about how observers categorize surfaces and areas in natural environments. Nevertheless the current research provides a demonstration and description of bias in spatial memory, and in doing so, our findings underscore the idea that motor and verbal estimates in at least some settings may differ more in degree than in kind.

\section{Acknowledgments}

The authors thank Robin K. Morris for helpful comments throughout the project and David Waller and Iris Trinkler for helpful discussions of earlier drafts.

Douglas H. Wedell's work on this research was supported in part by an NSF grant (SES-9911132). This research was also partly supported by European Union Marie Curie Fellowships, awarded to the first author through the Institute of Cognitive Neuroscience, University College London. The authors are solely responsible for information communicated and the European Commission is not responsible for any views or results expressed.

\section{References}

Allen, G. L. (1981). A developmental perspective on the effects of subdividing macrospatial experience. Journal of Experimental Psychology: Human Learning and Memory, 7, 120-132.

Allen, G. L., \& Haun, D. B. M. (2004). Proximity and precision in spatial memory. In G. Allen (Ed.), Human spatial memory: remembering where (pp. 41-63). Mahwah, NJ: Lawrence Erlbaum Associates.

Baddeley, A. (1986). Working memory. Oxford: Clarendon.

Bhalla, M., \& Proffitt, D. R. (1999). Visual-motor recalibration in geographical slant perception. Journal of Experimental Psychology: Human Perception and Performance, 25, 1076-1096.

Bhalla, M., \& Proffitt, D. R. (2000). Geographical slant perception: dissociation and coordination between explicit awareness and visually guided actions. In Y. Rossetti \& A. Revonsuo (Eds.), Dissociation but interaction between nonconscious and conscious processing. Amsterdam: John Benjamins.

Bridgeman, B., Peery, S., \& Anand, S. (1997). Interaction of cognitive and sensorimotor maps of visual space. Perception \& Psychophysics, 59(3), 456-469.

Burgess, N., Spiers, H. J., \& Paleologou, E. (in press). Orientational manoeuvres in the dark: dissociating allocentric and egocentric influences in spatial memory. Cognition.

Creem, S. H., \& Proffitt, D. R. (1998). Two memories for geographical slant: separation and interdependence of action and awareness. Psychonomic Bulletin and Review, 5, 22-36.

Creem, S. H., \& Proffitt, D. R. (1999). Separate memories for visual guidance and explicit awareness: the roles of time and place. In B. H. Challis \& B. N. Velichkovsky (Eds.), Stratification of consciousness and cognition (pp. 73-94). Amsterdam: John Benjamins Publishing.

Creem, S. H., \& Proffitt, D. R. (2001a). Grasping objects by their handles: a necessary interaction between cognition and action. Journal of Experimental Psychology: Human Perception and Performance, 27, $218-228$. 
Creem, S. H., \& Proffitt, D. R. (2001b). Defining the cortical visual systems: "what", "where", and "how". Acta Psychologica, 107, 43-68.

Diedrichsen, J., Werner, S., Schmidt, T., \& Trommershäuser, J. (2004). Immediate spatial distortions of pointing movements induced by visual landmarks. Perception \& Psychophysics, 66, 89-103.

Eby, D. W., \& Loomis, J. M. (1987). A study of visually directed throwing in the presence of multiple distance cues. Perception \& Psychophysics, 41, 308-312.

Goodale, M. A., \& Milner, A. D. (1992). Separate visual pathways for perception and action. Trends in Neurosciences, 15, 20-25.

Gravetter, F., \& Lockhead, G. R. (1973). Criterial range as a frame of reference for stimulus judgment. Psychological Review, 80, 203-216.

Hartley, T., Trinkler, I., \& Burgess, N. (2004). Geometric determinants of human spatial memory. Cognition, 94(1), 39-75.

Hollands, J. G., \& Dyre, B. P. (2000). Bias in proportion judgments: the cyclical power model. Psychological Review, 107, 500-524.

Huttenlocher, J., Hedges, L. V., \& Duncan, S. (1991). Categories and particulars: prototype effects in estimating spatial location. Psychological Review, 98, 352-376.

Lee, D. N. (1993). Body-environment coupling. In U. Neisser (Ed.), Ecological and interpersonal sources of self-knowledge (pp. 43-67). Cambridge, UK: Cambridge University Press.

Link, S. W. (1992). The wave theory of difference and similarity. Hillsdale, NJ: Lawrence Erlbaum Associates.

Logie, R. (1995). Visuo-spatial working memory. Hove, England: Lawrence Erlbaum Associates.

Loomis, J. M., Silva, J. A., Fujita, N., \& Fukusima, S. S. (1992). Da Visual space perception and visually directed action. Journal of Experimental Psychology: Human Perception and Performance, 18, 906-921.

Luce, R. D., Nosofsky, R. M., Green, D. M., \& Smith, A. F. (1982). The bow and sequential effects in absolute identification. Perception \& Psychophysics, 32, 397-408.

McNamara, T. P., Rump, B., \& Werner, S. (2003). Egocentric and geocentric frames of reference in memory of large-scale space. Psychonomic Bulletin and Review, 10, 589-595.

Milner, A. D., \& Goodale, M. A. (1995). The visual brain in action. New York: Oxford University Press.

Montello, D. R., Richardson, A. E., Hegarty, M., \& Provenza, M. (1999). A comparison of methods for estimating directions in egocentric space. Perception, 28, 981-1000.

Mou, W., \& McNamara, T. P. (2002). Intrinsic frames of reference in spatial memory. Journal of Experimental Psychology: Learning, Memory, and Cognition, 28, 162-170.

Neggers, S. F. W., Schölvinck, M. L., van der Lubbe, R. H. J., \& Postma, A. (2005). Quantifying the interactions between allo- and egocentric representations of space. Acta Psychologica, 118, 25-45.

O'Keefe, J., \& Nadel, L. (1978). The hippocampus as a cognitive map. Oxford, UK: Oxford University Press.

Parslow, D. M., Morris, R. G., Fleminger, S., Rahman, Q., Abrahams, S., \& Recce, M. (2005). Allocentric spatial memory in humans with hippocampal lesions. Acta Psychologica, 118, 123-147.

Philbeck, J. W., \& Loomis, J. M. (1997). Comparison of two indicators of visually perceived egocentric distance under full-cue and reduced-cue conditions. Journal of Experimental Psychology: Human Perception and Performance, 23, 72-85.

Postma, A., Kessels, R. P. C., \& van Asselen, M. (2004). The neuropsychology of object-location memory. In G. Allen (Ed.), Human spatial memory: remembering where (pp. 143-160). Mahwah, NJ: Lawrence Erlbaum Associates.

Proffitt, D. R., Bhalla, M., Gossweiler, R., \& Midgett, J. (1995). Perceiving geographical slant. Psychonomic Bulletin and Review, 2, 409-428.

Proffitt, D. R., Stefanucci, J., Banton, T., \& Epstein, W. (2003). The role of effort in perceiving distance. Psychological Science, 14, 106-112.

Rossetti, Y. (1998). Implicit short-lived motor representations of space in brain damaged and healthy subjects. Consciousness and Cognition, 7, 520-558.

Stevens, A., \& Coupe, P. (1978). Distortions in judged spatial relations. Cognitive Psychology, 10, 422 437. 
Waller, D., Loomis, J. M., Golledge, R. G., \& Beall, A. C. (2000). Place learning in humans: the role of distance and direction information. Spatial Cognition and Computation, 2, 333-354.

Werner, S., \& Diedrichsen, J. (2002). The time course of spatial memory distortions. Memory \& Cognition, $30,718-730$.

Wilkinson, L. (1989). SYSTAT: The system for statistics. Evanston, IL: SYSTAT, Inc.

Wraga, M., Creem, S. H., \& Proffitt, D. R. (2000). Perception-action dissociations of a walkable MullerLyer configuration. Psychological Science, 11, 239-243. 